\title{
Incidence of vaginal Weeksella virosa (formerly
} group IIf )

\author{
C MARDY, * B HOLMES $\dagger$
}

From the *Department of Pathology, The General Hospital, Altrincham, Cheshire, and the †Computer Identification Laboratory, National Collection of Type Cultures, Central Public Health Laboratory, London

SUMMARY The antimicrobial susceptibilities, biochemical properties, and cultural characteristics of six strains of Weeksella virosa (formerly group IIf) were determined. The main characteristics of this non-fermentative organism were production of cytochrome oxidase, gelatinase, and indole, but a lack of saccharolytic activity.

A study was then made of the isolation rates of Weeksella virosa from high vaginal swabs from 300 women: a healthy control group $(n=100)$, a general group with symptoms of vaginal infection $(n=100)$, and 100 women from a remand centre, where likelihood of exposure to sexually transmitted disease might be expected to be higher. The incidence of Weeksella virosa was found to be $2 \%$ in the first two groups. This suggests that, irrespective of the presence of pathogens, the incidence of the species in the general female population is around $2 \%$. A much higher incidence (15\%) was found in the third group, suggesting sexual transmission of the organism.

In 1970 Pickett and Manclark described eight strains which they regarded as non-saccharolytic flavobacteria.' They reported the biochemical characteristics of the strains ${ }^{23}$ and their susceptibility to antimicrobial agents, ${ }^{4}$ but they did not consider any of them to be pathogenic. Twelve similar strains were reported by Olsen and $\operatorname{Ravn}^{5}$; they were isolated predominantly from female genital tract specimens submitted for examination for Neisseria gonorrhoeae. Owen and Snell examined eight such strains, five were from the urethra and one each from the cervix and vagina ${ }^{6}$; they found the properties of the strains to be intermediate between those of Flavobacterium and Moraxella. Tatum et al described 78 strains and designated them as group IIf ${ }^{7}$; they recently reported 87 strains primarily from urine $(45 \%)$, the cervix $(15 \%)$, and the vagina $(15 \%){ }^{8} \mathrm{~A}$ single group IIf strain, from the vagina of an asymptomatic postmenopausal woman, has also been reported.' A new genus and species, Weeksella virosa, has now been proposed for the group. $^{10}$

In the pathology department at Altrincham General Hospital an unidentified non-fermentative Gram negative rod was occasionally seen on selective chocolate blood agar plates being used for the isolation of Neisseria gonorrhoeae from high vaginal swabs. Representative strains of this unidentified organism

Accepted for publication : + September 1987 submitted to the Computer Identification Laboratory of the National Collection of Type Cultures (NCTC) were identified as $W$ virosa. Six $W$ virosa strains were selected from a number of such strains isolated from high vaginal swabs and characterised at the hospital in a wide range of biochemical tests, in the API $20 \mathrm{E}$ system, and in the Roche Oxi/Ferm tube.

A study was made of the rates of isolation of Weeksella virosa from the high vaginal swabs of three different groups of women. Group 1 comprised well women showing no signs of bacterial infection; this group consisted mainly of volunteers and those undergoing routine preoperative high vaginal swabs. Group 2 comprised patients referred for investigation due to symptoms of possible vaginal infection. Group 3 comprised women from a special source (women's remand centre) who were possibly at greater risk of contracting sexually transmitted disease (STD). One hundred specimens were sampled from each group.

\section{Material and methods}

Charcoal swabs, transported in Stuart's medium (Medical Wire STMW 219) were used for the collection of high vaginal specimens. Selective chocolate blood agar containing colistin sulphate $(7.5 \mu \mathrm{g} / \mathrm{ml})$, trimethoprim $(5 \mu \mathrm{g} / \mathrm{ml})$, and vancomycin $(3 \mathrm{U} / \mathrm{ml})$ was used routinely for the isolation of Neisseria gonorrhoeae. The presence of antibiotics in the media ensures 
the suppression of normal vaginal flora ( $W$ virosa grows well on this medium as it is resistant to all three antibiotics at these concentrations).

Initially a smear was prepared on a sterile slide, and each high vaginal swab was subcultured on to nonselective chocolate $(10 \% \mathrm{w} / \mathrm{v})$ blood agar, selective chocolate $(10 \% \mathrm{w} / \mathrm{v})$ blood agar, and finally into Trichomonas medium (Medical Wire TMMW 220). The smear was fixed and stained by Gram's method (Lillie's modification). " The Trichomonas medium was incubated for 48 hours at $37^{\circ} \mathrm{C}$ aerobically; both chocolate blood agar plates were incubated in an atmosphere of about $8-10 \%(\mathrm{v} / \mathrm{v})$ carbon dioxide for 48 hours.

\section{CULTURAL CHARACTERISTICS}

Commercially poured agar plates were used (Analytical and Bacteriological Media, London). The six strains chosen for detailed study were inoculated on to $10 \%(\mathrm{w} / \mathrm{v})$ horse blood agar, $10 \%(\mathrm{w} / \mathrm{v})$ selective chocolate horse blood agar, MacConkey agar and nutrient agar. The blood agar plates were incubated at $4^{\circ} \mathrm{C}, 22^{\circ} \mathrm{C}, 37^{\circ} \mathrm{C}$ and $43^{\circ} \mathrm{C}$. Smears were made from colonies of each strain and stained by Gram's method."

\section{CONVENTIONAL BIOCHEMICAL TESTS}

All tests were recorded at or up to seven days' incubation, except where stated. The following tests were carried out mainly by the media and methods described by Cowan and Steel ${ }^{11}$ : cytochrome oxidase production (one day); acid from carbohydrates (method 1); oxidation or fermentation (or alkali production) in glucose O-F medium; citrate utilisation (method 1); dihydrolase for arginine and decarboxylases for lysine and ornithine (method 1$) ; \mathrm{H}_{2} \mathrm{~S}$ production (method 1); indole production (methods 2 and 3 ), potassium cyanide tolerance (two days); production of acetylmethylcarbinol (Voges-Proskauer test, Baritt's method); $\beta$-galactosidase production (two days); and urease production (method 1). The following tests were carried out by the specific methods indicated: catalase production,,$^{12}$ methyl red reaction,,$^{13}$ nitrate reduction, ${ }^{14}$ phenylalanine deamination ${ }^{15}$ and gelatinase production. ${ }^{16}$ Pigment production was examined daily for five days on nutrient agar and on $1 \%(w / v)$ tyrosine agar.

\section{A PI 20E TESTS}

Each of the six strains was inoculated into an API $20 \mathrm{E}$ strip and incubation for 48 hours was required in accordance with the manufacturer's instructions; the six supplementary tests required were also performed. The nine digit profile number thus obtained was consulted in the API 20E Analytical Prafile Index (July 1985 edition). Any additional tests required by the API
$20 \mathrm{E}$ system to further the identification were also performed.

\section{OXI/FERM TUBES}

In accordance with the manufacturer's instructions the six strains were inoculated into Oxi/Ferm tubes; after incubation for 48 hours indole reagent was added $\frac{\bar{p}}{\overline{ }}$ to the appropriate test compartment. The profile $\stackrel{\mathbb{\Omega}}{\Omega}$ number obtained was consulted in the Oxi/Ferm tuben identification scheme (CCIS system 1979-1432) and any supplementary tests required to further the identification were performed.

\section{ANTIMICROBIAL SUSCEPTIBILITY}

Tests were performed by inoculating blood agar platesi on a rotary plater from four to six hour broth cultures. $\vec{\sim}$ After antibiotic discs were added the plates weren incubated at $37^{\circ} \mathrm{C}$ for 18 hours. The following antibiotic discs were used: ampicillin $(10 \mu \mathrm{g})$; chloram-윽 phenicol $(25 \mu \mathrm{g})$; colomycin $(25 \mu \mathrm{g})$; erythromycin$(5 \mu \mathrm{g})$; kanamycin $(30 \mu \mathrm{g})$; neomycin $(10 \mu \mathrm{g}) ;{ }_{\mathbb{\infty}}^{\top}$ novobiocin $(5 \mu \mathrm{g})$; penicillin $\mathrm{G}(1 \mathrm{U})$; streptomycino $(25 \mu \mathrm{g})$; tetracycline $(25 \mu \mathrm{g})$; Escherichia coli NCTC 10418 was used as a control organism.

\section{ISOLATION STUDY}

The same procedure as for the routine examination of high vaginal swabs was followed except that high vaginal swabs from group 1 were also subjected to this procedure; each high vaginal swab was alson subcultured on to a $10 \%(\mathrm{w} / \mathrm{v})$ blood agar plate which $\overline{\mathrm{D}}$ was incubated for 48 hours at $37^{\circ} \mathrm{C}$ aerobically. $\stackrel{\varrho}{\Rightarrow}$ Colonies suspected of being $W$ virosa were examinedo for cytochrome oxidase production and by $\mathrm{Gram}_{\supset}^{3}$ stain. Colonies giving the reactions expected for $W \frac{\mathrm{F}}{\mathrm{O}}$ virosa had their identities confirmed by the API 20E system.

\section{Results}

CULTURAL CHARACTERISTICS

After 18 hours of incubation on $10 \%(w / v)$ blood agar음 all six strains appeared as cream-coloured, moist $>$ domed colonies (1-2 mm in diameter). On furthes. incubation the colonies became slightly larger and very glossy. A pale $\alpha$-haemolysis was noted after $3 \sigma^{\circ}$ hours of incubation which intensified when the plates were left at room temperature. Growth on non-selec $N$ tive and selective chocolate blood agar was mucto better than on ordinary blood agar-very moisto cream-coloured colonies $2-3 \mathrm{~mm}$ in diameter were obtained after 18 hours. On further incubation the colonies became confluent, making subsequent sub-0 culture on to further chocolate blood agar plates tơ? obtain single colonies very difficult, as confluen growth was obtained however small the inoculum. The्ष्ट 


\section{Table 1 Biochemical characteristics of Weeksella virosa}

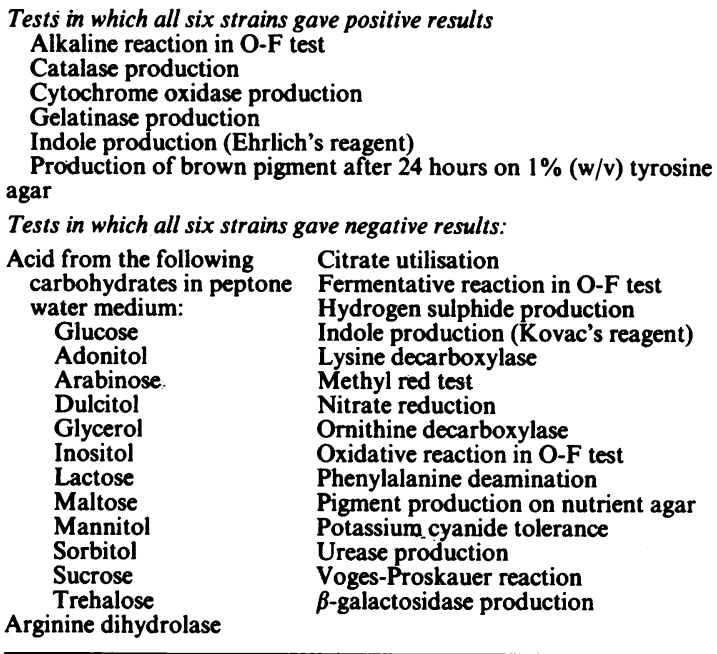

Table 2 Isolation rates in different groups of women

\begin{tabular}{lll}
\hline $\begin{array}{l}\text { Group No } \\
\text { (100 patients } \\
\text { in each group) }\end{array}$ & $\begin{array}{l}\text { No of isolations of } \\
\text { Weeksella virosa }\end{array}$ & $\begin{array}{l}\text { No of specimens in } \\
\text { which a pathogen was } \\
\text { also isolated }\end{array}$ \\
\hline 1 & 2 & 0 \\
2 & 2 & 2 \\
3 & 15 & 5 \\
\hline
\end{tabular}

six strains produced luxurious growth on nutrient agar and the growth was seen to flow down nutrient agar slopes, collecting in the bottom of the bilou jar. No growth was obtained on MacConkey agar. Growth was obtained on $10 \%(\mathrm{w} / \mathrm{v})$ blood agar and in nutrient broth at $22^{\circ} \mathrm{C}, 37^{\circ} \mathrm{C}$, and $43^{\circ} \mathrm{C}$. No growth was seen in either medium at $4^{\circ} \mathrm{C}$. In a hanging drop suspension prepared from the nutrient broths at $22^{\circ} \mathrm{C}$ and $37^{\circ} \mathrm{C}$, the strains showed no motility. In Gram stained films from blood agar the organisms appeared as large (about 3-5 $\mu \mathrm{m}$ in length), Gram negative rods with rounded ends; some short cocco-bacillary forms were also seen. In the broth cultures the cells tended to form long filaments.

The results of the biochemical tests are given in table 1 .

\section{API 20E TESTS}

After 48 hours of incubation in the API 20E system all six strains yielded the profile number 0002004-00. This number is listed in the API $20 E$ Analytical Profile Index with the comment "low discrimination", but the most likely taxon is CDC group IIf, followed by Moraxella spp. The results of four additional tests (acetate utilisátion, growth at $42^{\circ} \mathrm{C}$, growth on MacConkey agar and pigment production) allowed the strains to be ascribed to CDC group IIf using the appropriate table in the API 20E Profile Index.

\section{OXI/FERM TUBES}

An "ID value" of 0201 was obtained from the Roche Oxi/Ferm tube results for all six strains after 48 hours of incubation. The "ID value" in the CCIS system listed three possible taxa, but performance of the five supplementary tests (alkaline reaction in xylose, penicillin susceptibility, production of brown pigment, production of yellow pigment and "runny growth") permitted identification of the strains as "group $2 \mathrm{~F}$, Flavobacterium-like."

\section{ANTIMICROBIAL SUSCEPTIBILITY}

All six strains were resistant to kanamycin, neomycin, and trimethoprim; the strains were susceptible to ampicillin, chloramphenicol, colomycin, erythromycin, novobiocin, penicillin $\mathrm{G}$, streptomycin, and tetracycline.

\section{ISOLATION STUDY}

The isolation rates of Weeksella virosa are given in table 2. A bigher incidence of Weeksella virosa was seen in the group of women from the remand centre than in the two other groups. The presence of common pathogens and the results of the Gram stained high vaginal smears were noted for any patient from whom Weeksella virosa was isolated. The pathogens included Candida albicans, Neisseria gonorrhoeae, and Trichomonas vaginalis. No clinically important information could be formulated from the Gram stained smears, most of which contained large numbers of normal flora: Gram positive bacilli, Gram negative rods, Gram positive cocci, and in some cases, a presumptive pathogen such as yeast cells. In most smears of patients from whom Neisseria gonorrhoeae had been isolated, the normal flora was reduced.

\section{Discussion}

Few reports on the incidence of Weeksella virosa in human clinical specimens have been published. Tatum et al identified only 78 cultures as group IIf from among 35000 cultures received for identification between 1949 and 1973. ${ }^{7}$ The incidence of this species in clinical material seems to be low, but the results of the present study suggest that the incidence of the species is higher and that the organism is probably ignored in most routine hospital laboratories, especially when isolated from clinical specimens containing a high proportion of normal flora. The mucoid nature of the colonies would often cause them to be mistakenly regarded as klebsiellas. 
The clinical importance of the presence of $W$ virosa is difficult to assess from the results of the isolation rate study. In group $2 W$ virosa was isolated only when a pathogen was present. This might suggest that the organism is normally suppressed in a healthy patient but becomes prominent when a patient is immunocompromised. The same isolation rate for $W$ virosa, however, was obtained in control group 1, suggesting that irrespective of the presence of pathogens the incidence of $W$ virosa in the general female population is around $2 \%$. Group 3 probably included some women with a lot of sexual partners and therefore passage of $W$ virosa might be increased. This could account for the higher isolation rate in this group (15\%) compared with groups 1 and 2 . Interestingly, in group 3 although the incidence of pathogens present in the same patient from whom $W$ virosa was isolated, was higher $(5 \%)$ than that of group $2(2 \%)$, the increase was not as high as that seen for $W$ virosa $(15 \%)$.

$W$ virosa is apparently much more common in women than in men, with the highest isolation rates from urine and the genital or urethral tract. ${ }^{810}$ In this study all isolations of the organism were from the female genital tract. It would be interesting to know if a link between $W$ viros $a$ and sexual transmission could be proved, but this would require a much more extensive study (including groups of male patients), perhaps on a national scale.

$W$ virosa is isolated with apparent ease from vaginal specimens and may be identified with a minimum amount of labour and equipment, using either the API $20 \mathrm{E}$ system or the Roche Oxi/Ferm tube. The conventional biochemical test results given in table 1 agree with those given in the original description of the species. ${ }^{10}$ Although almost all non-fermenters also fail to exhibit acid production in a peptone water medium, $W$ virosa strains are also non-saccharolytic in ammonium salt sugar medium, which is generally more appropriate for showing acid production from carbohydrates by non-fermenters. ${ }^{10}$ Indole production by $W$ virosa strains is characteristic, but the results clearly show that a sensitive technique is necessary.

Flavobacterium species are highly resistant organisms but $W$ virosa has been reported to be much more susceptible, even to penicillin, with resistance shown only to kanamycin and tobramycin. ${ }^{17} \mathrm{~W}$ virosa has been shown to be susceptible to all $\beta$-lactam producing antibiotics tested, as well as to aztreonam, chloramphenicol, and clindamycin. ${ }^{41819}$ The results of the present study agree with these findings.

We thank Dr R Jennings and Miss A J Kelly for their invaluable support.

\section{References}

1 Pickett MJ, Manclark CR. Nonfermentative bacilli associated 은 with man: I. Nomenclature. Am J Clin Pathol 1970;54:155-63. $\Rightarrow$

2 Pickett MJ, Pedersen MM. Nonfermentative bacilli associated $\widehat{D}$ with man: II. Detection and identification. Am J Clin Patholo 1970a;54:164-77.

3 Pickett MJ, Pedersen MM. Salient features of non-saccharolytic $\frac{\bar{\sigma}}{\bar{\omega}}$ and weakly saccharolytic nonfermentative rods. Can $J \frac{\Phi}{\vec{T}}$ Microbiol 1970b;16:401-9.

4 Pedersen MM, Marso E, Pickett MJ. Nonfermentative bacilli associated with man: III. Pathogenicity and antibiotic suscep- $\backsim$ tibility. Am J Clin Pathol 1970;54:178-92.

5 Olsen H, Ravn T. Flavobacterium meningosepticum isolated from the genitals. Acta Pathol Microbiol Scand (Sect B) 1971;79: $\vec{\omega}$ 102-6.

6 Owen RJ, Snell JJS. Comparison of group IIf with Flavobacterium and Moraxella. Antonie van Leeuwenhoek 1973;39:? 473-80.

7 Tatum HW, Ewing WH, Weaver RE. Miscellaneous Gram- $\vec{i}$ negative bacteria. In: Lennette EH, Spaulding EH, Truant JP, N eds. Manual of clinical microbiology. 2nd ed. Washington, DC: $\underset{-}{-}$ American Society for Microbiology, 1974:270-94.

8 Clark WA, Hollis DG, Weaver RE, Riley P. Identification of $\frac{0}{2}$ unusual pathogenic Gram-negative aerobic and facultatively anaerobic bacteria. Atlanta: US Department of Health and $T$ Human Services, 1984.

9 Tashjian JH, Coulam CB, Washington II JA. Vaginal flora in asymptomatic women. Mayo Clin Proc 1976;51:557-61.

10 Holmes B, Steigerwalt AG, Weaver RE, Brenner DJ. Weeksella virosa gen. nov, sp. nov. (formerly group IIf), found in human $\vec{\theta}$ clinical specimens. System Appl Microbiol 1986;8:185-90.

11 Cowan ST, Steel KJ. Manual for the identification of medical. bacteria. Cambridge: Cambridge University Press, 1965.

12 Deibel RH, Evans JB. Modified benzidine test for the detection of cytochrome-containing respiratory systems in microorganisms. J Bacteriol 1960;79:356-60.

13 Clark WM, Lubs HA. The differentiation of bacteria of the colonaerogenes family by the use of indicators. J Infect Dis 1915; 17:160-73.

14 Cook GT. A plate test for nitrate reduction. J Clin Pathol $\overrightarrow{\bar{\sigma}}$ 1950;3:359-62.

15 Ewing WH, Davis BR, Reavis RW. Phenylalanine and malonate $\overline{-}$ media and their use in enteric bacteriology. Public Health Laboratory 1957;15:153-67.

$16 \mathrm{Kohn} \mathrm{J.} \mathrm{A} \mathrm{preliminary} \mathrm{report} \mathrm{of} \mathrm{a} \mathrm{new} \mathrm{gelatin} \mathrm{liquefaction}$ method. J Clin Pathol 1953;6:249.

17 Von Graevenitz A. Clinical significance and antimicrobial susceptibility of flavobacteria. In: Reichenbach $\mathrm{H}$, Weeks OB, eds. The Flavobacterium-Cytophaga group. Proceedings of the inter-尺ิ national symposium on yellow-pigmented Gram-negative bac- 3 teria of the Flavobacterium Cytophaga group, Braunschweig July 8th to 11 th, 1980. Weinheim: Verlag Chemie, 1981:153-64. 윽

18 Fass RJ, Barnishan J. In vitro susceptibilities of non-fermentative $D$ Gram-negative bacilli other than Pseudomonas aeruginosa to음 32 antimicrobial agents. Rev Infect Dis 1980;2:841-53.

19 Schell RF, Francisco M, Bihl JA, LeFrock JL. The activity of ceftazidime compared with those of aztreonam, newer cephalosporins and Sch 29482 against nonfermentative Gramnegative bacilli. Chemotherapy 1985;31:181-90.

Requests for reprints to: Dr B Holmes, NCTC, Centra? Public Health Laboratory, 61 Colindale Avenue, Londono NW9 5HT, England. 